\title{
Effects of Probiotics with or without Honey on Radiation-induced Diarrhea
}

\author{
H.S. Mansouri-Tehrani ${ }^{1}$, M. Rabbani Khorasgani ${ }^{2}$, M. Roayaei $^{*}$ \\ ${ }^{1}$ Department of Biotechnology, University of Isfahan, Isfahan, Iran \\ ${ }^{2}$ Department of Biology, University of Isfahan, Isfahan, Iran \\ ${ }^{3}$ Department of Radiation Oncology, Isfahan University of Medical Sciences, Isfahan, Iran
}

\section{- Original article}

\section{*Corresponding author:}

Dr. Mahnaz Roayaei,

Fax: +983132337964

E-mail: Roayaei@med.mui.ac.ir

Revised: Sept. 2015

Accepted: Oct. 2015

Int. J. Radiat. Res., July 2016;

14(3): 205-213

DOI: 10.18869 /acadpub.ijrr.14.3.205

\section{ABSTRACT}

Background: Diarrhea is a well-recognized side effect associated with pelvic radiation; however, there is not any effective common treatment for radiation-induced diarrhea. A popular alternative is probiotics, which have been used in several gastrointestinal disorders. Probiotics are live microbial food supplements. Furthermore, honey is a putative nutritional with a variety of health effects, including antibacterial, antioxidant, anti-inflammatory and prebiotic. The present study evaluated the effects of probiotic with or without honey on radiation-induced diarrhea. Materials and Methods: Sixty-seven adult patients with pelvic cancer underwent radiotherapy for four weeks. They randomized to receive probiotic $(n=22)$, probiotic plus honey $(n=21)$ or placebo $(n=24)$ from one week before radiotherapy for five weeks. Diarrhea grade and stool consistency score were recorded weekly according to the Common Toxicity Criteria system and the Bristol scales, respectively. Results: The results showed a decrease in the daily number of bowel movements $(p=$ 0.003 and 0.006$)$, diarrhea grade $(p=0.001$ and 0.001$)$ and the need for antidiarrheal medication ( $p=0.021$ and 0.041 ) also an increase in the stool consistency ( $p=0.004$ and 0.005 ) in patients who either used probiotic or probiotic plus honey (respectively), these were significant in weeks 4 and 5 of treatment. Conclusion: Probiotics with or without honey can reduce the incidence of radiation-induced diarrhea and the need for antidiarrheal medication.

Keywords: Probiotics, honey, diarrhea, radiotherapy, pelvic cancer.

\section{INTRODUCTION}

Pelvic radiotherapy, either alone or with chemotherapy, has established to be successful in the treatment of many of pelvic cancers. Pelvic radiation causes acute intestinal symptoms in over $80 \%$ of patients include diarrhea, nausea, and vomiting and reduces quality of life in about $50 \%$ of patients (1-3). The main adverse event of pelvic radiation is diarrhea (4-7). Cancer patients suffering from diarrhea complain of feeling fear, shame, and distress $(5,8)$.

There is a large intestinal mucosal in the pelvic radiation field. Symptoms usually begin during the second week of treatment $(3,6,9,10)$ and can cause other problems such as malnutrition, abdominal pain, fecal incontinence, proctorrhagia, dehydration, weakness, stress (3, 11-14) and the function of the immune system is reduced (15).

There are no prophylactic agents confirmed for the prevention of pelvic radiation enteritis (14, 16). Some of nutritional regimes have been tried, but evidences are weak and limited $(17,18)$. Radiation induced diarrhea is treated with medication such as Loperamide $(19,20)$. Treatment failure occurs in many patients $(20,21)$. The new methods that aim at other mechanisms in the pathophysiology of radiation-induced diarrhea 
are required. Recently, probiotics are well known to have beneficial effects on intestinal problems (22-25). Studies in animal models and clinical trials of patients with inflammatory bowel disease (IBD) have frequently shown that use of probiotic organisms can effectively modulate the severity of intestinal inflammation through altering the composition and the metabolic and functional properties of gut flora $(26,27)$. Some clinical studies have investigated the use of probiotics for the prevention and treatment of radiation induced diarrhea $(19,21,28-$ 32). Probiotic literally means "for life". Probiotics are live microorganisms when ingested in sufficient numbers, they are expected to confer one or more proven health benefits on the consumer. Probiotics are supplements including viable nonpathogenic microorganisms such as Lactobacillus and Bifidobacterium to modulate the host's microflora by colonization and maintenance of the balance between proinflammatory and anti-inflammatory cytokines (10, 33-35). Probiotics function has actually remained unclear, although several hypotheses are being proposed. Probiotics may facilitate the production of antibacterial substances (cytokines and butyric acid), reduce the pathogenic species by creating competitive inhibition, decrease epithelial permeability to intraluminal pathogens, stimulate immune cells and create a barrier to pathogens by lowering intestinal $\mathrm{pH}$. They also stimulate lactase production, which helps lactose digestion, and enhance intestinal micro flora (22-24,36-38).

Abdominal bloating may occur in some probiotic users and occurrence of bloating may be resulted from gas production in the gastrointestinal (39).

'Prebiotics' are food ingredients that stimulate the growth and/or activity of probiotics (40). Honey properties are antiinflammatory, antioxidant, antitumor (41-43) and effective 'prebiotic' (44-46). On the other hand, according to an old belief, patients with diarrhea to improve their problem eat yogurt (47). Also yogurt is known as a popular food carrier of probiotics (48).

There are few experiences in effect of probiotic organisms for the prevention and treatment of radiation-induced diarrhea in cancer patients and probiotic plus honey has not been used for this purpose yet. The primary objective of this study was to evaluate the efficacy of the probiotic with or without honey in comparison with placebo, to prevent or delay the incidence of radiation-induced diarrhea. Secondary objectives were to assess if intake of probiotic with or without honey decreases the need of antidiarrheal medication as well as increases in stool consistency. Overall the main aim of research was to find more effective and with less side effect regimen for control of radiation-induced diarrhea.

\section{MATERIALS AND METHODS}

A randomized, Placebo-Controlled Study was performed on 67 patients diagnosed with pelvic cancers (colorectal, prostate, endometr, bladder, ovary, cervix, bone sarcoma) that were referred to Radiation Oncology Clinic at Sayyed-AlShohada Hospital, Isfahan, Iran from October 2012 to May 2013. The treatment protocol was explained to patients and they were given informed consent to participate. Exclusion criteria included opioid usage, antimicrobial treatment and the presence of any acute or chronic gastrointestinal condition associated with diarrhea for at least one month before recruitment. Study protocol, the informed consent and materials were reviewed and approved by the Ethical Committee of the Isfahan University of Medical Sciences. This study was registered in the Iranian Registry of Clinical Trials (IRCT2015030421338N1).

All the patients were irradiated with conventional radiotherapy to a total dose from 4000 to 5000 cGy (1.8 Gy/day) with $18 \mathrm{MV}$. The upper edge of the AP field varied from the third lumbar vertebra to the first sacral bone and extended to the lower edge of the pubic bones and laterally, covering a $1.5-2 \mathrm{~cm}$ of the pelvic inlet rim, varied according to the abdominopelvic disease. Patients received conventional radiotherapy five fractions weekly for 4 -5 weeks. 
Probiotic capsules 'LactoCareò’ (Zist Takhmir Company, Tehran, Iran) contained: Lactobacillus casei $1.5 \times 10^{9} \mathrm{CFU}$, Lactobacillus acidophilus 1.5 $\times 10^{10} \mathrm{CFU}$, Lactobacillus rhamnosus $3.5 \times 10^{9}$ CFU, Lactobacillus bulgaricus $2.5 \times 10^{8} \mathrm{CFU}$, Bifidobacterium breve $1 \times 10^{10} \mathrm{CFU}$, Bifidobacterium longum $5 \times 10^{8} \mathrm{CFU}$ and Streptococcus thermophilus $1.5 \times 10^{8} \mathrm{CFU}$ per 500 mg. Coriander honey was obtained from Zagros Mountains (Khansar) in Iran (Keshtzare Sabz Company, Khansar, Iran). Fresh honey bee used in this study was assessed and approved by the Food and Drug Administration Isfahan University of Medical Sciences, according to Iranian National Standard No.92. Placebo capsules containing $500 \mathrm{mg}$ of corn starch, with the same size, shape and color of probiotic capsules were purchased from Zist Takhmir Company, Tehran, Iran. Yogurt In packages of $150 \mathrm{~g}$ was purchased from Allas Dairy Company, Isfahan, Iran.

Simple randomization was used to allocate patients to three groups: group one received probiotics (two probiotic capsules per day, one capsule in the morning after breakfast and another one in the evening, after consuming 150 grams of low-fat yogurt); group two took a combination of probiotic and honey (two probiotic capsules and 30 grams honey per day, one capsule and 15 grams honey in the morning after breakfast and another one capsule in the evening, after consuming 150 grams of low-fat yogurt and 15 grams honey at night); group three was the control group received two placebo capsules per day, one capsule in the morning after breakfast and another one in the evening, after consuming 150 grams of low-fat yogurt). After randomization, the patients underwent our treatment one week before starting pelvic radiotherapy and then they continued to take the same their regime during the treatment course (in total 5 weeks).

Equal dietary recommendations, including a list of prohibited and allowed foods, were given to all the participants. All patients received the same kind and rate of low-fat yogurt and consumption of other dairy products except yogurt were not allowed. Taking antidiarrheal medication (except prescribed by the physician) and antimicrobial drug were also prohibited; however, if patients needed to take antidiarrheal medication its consumption was recorded as an efficacy criterion.

Severity of the diarrhea was evaluated according to the Common Toxicity Criteria of the National Cancer Institute (49): grade $1=$ increase of 2-3 stools per day compared to pre-treatment, grade 2 = increase of 4- 6 stools per day or nocturnal stools, grade $3=$ increase of 7-9 stools per day or incontinence, grade $4=$ increase of 10 or more stools, IV hydration needed. To evaluate the consistency of stool, adapted Bristol scale (50) was used to facilitate its use by patients: normal stools (Bristol 1-4), soft stools, in pieces (Bristol 5-6) and liquid stools, no shape (Bristol 7). The patients evaluated for the daily number of bowel movement (defecation), diarrhea grade, stool consistency score, the need for antidiarrheal medication and bloating weekly by one person.

Data were analyzed using: Chi-square, Fisher's exact and one-way ANOVA test. Post analysis was done by LSD (Least Significant Difference) method in order to do paired comparison (treatment group VS. placebo group). The pvalue less than 0.05 was considered as statistically significant. Statistical analyses were performed using SPSS statistical software (v. 16, SPSS Inc., Chicago, IL, USA).

\section{RESULTS}

Among 78 patients involved in this study, 11 patients were excluded for failure to follow up. Patients were aged between 20 to 85 years (mean age $=62 \pm 14.8$ years). Patient characteristics are detailed in table 1.

\section{Daily number of bowel movements}

The minimum and maximum daily number of bowel movements was 0 and 7 for the probiotic group, and 0 and 10 for the probiotic plus honey and placebo groups respectively. The daily number of bowel movements was higher in week 4 for the probiotic (2.8) and placebo (4.9) groups and weeks 2 and 5 for the probiotic plus honey group (2.5). The results of the linear

Int. J. Radiat. Res., Vol. 14 No. 3, July 2016 


\section{Mansouri-Tehrani et al. / Probiotics and radiation-induced diarrhea}

mixed model test showed that the mean daily number of bowel movements in the placebo group was significantly higher than the probiotic and probiotic plus honey groups throughout the treatment course $(\mathrm{p}=0.003$ and $\mathrm{p}=0.006$ respectively) (figure 1).

\section{Diarrhea grade}

Moderate to severe diarrheal symptoms (grades 2, 3) was recorded in 31 (46.3\%) patients during pelvic radiotherapy. The number (percentage) patients with diarrhea grades 2 and 3 was 7 (31.8), 4 (19) and 17 (70.8) during the treatment for the probiotic, probiotic plus honey and placebo groups respectively. The results of Mann-Whitney test showed that the mean diarrhea grade in weeks 4 and 5 was significantly higher in the placebo group than the probiotic and probiotic plus honey groups $(\mathrm{p}=$ 0.007 and 0.001 for probiotic and $\mathrm{p}<0.001$ and $\mathrm{p}=0.001$ for probiotic plus honey in weeks 4 and 5 respectively) as shown in figure 2. Fisher's exact test also revealed that patients in the placebo group were diagnosed with grade 2 diarrhea, significantly, earlier than the probiotic $(\mathrm{P}=0.016)$ and probiotic plus honey groups $(\mathrm{P}=0.005)$.

Table 1. Patient characteristics.

\begin{tabular}{|c|c|c|c|c|}
\hline & \multicolumn{3}{|c|}{ Study arms } & \\
\hline & Probiotic & Probiotic- Honey & Placebo & \\
\hline & $(n=22)$ & $(n=21)$ & $(n=24)$ & $P$ \\
\hline Age (mean $\pm S D$ ) & $63.73 \pm 15.09$ & $57.86 \pm 17.48$ & $64.17 \pm 11.69$ & $.44^{\mathrm{a}}$ \\
\hline Gender & & & & $.15^{b}$ \\
\hline male & $14(20.9)$ & $8(11.9)$ & $17(25.4)$ & \\
\hline female & $8(11.9)$ & $13(19.4)$ & $7(10.4)$ & \\
\hline Cancer type & & & & $.42^{c}$ \\
\hline colorectal & $6(9.0)$ & 9 (13.4) & $9(13.4)$ & \\
\hline prostate & $6(9.0)$ & $3(4.5)$ & $6(9.0)$ & \\
\hline endometrial & $3(4.5)$ & $5(7.5)$ & $2(3.0)$ & \\
\hline bladder & $4(6.0)$ & 0 & $4(6.0)$ & \\
\hline ovary & $2(3.0)$ & $1(1.5)$ & $1(1.5)$ & \\
\hline cervical & $1(1.5)$ & $1(1.5)$ & $2(3.0)$ & \\
\hline bone sarcoma & 0 & $2(3.0)$ & 0 & \\
\hline ChRt (yes) & $8(11.9)$ & $9(13.4)$ & $9(13.4)$ & $.9^{b}$ \\
\hline
\end{tabular}

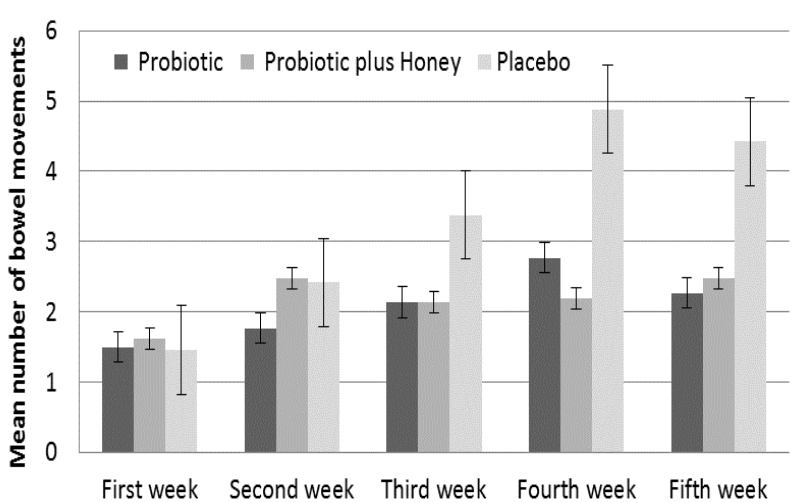

Figure 1. Comparison of the mean number of bowel movements per week during pelvic radiotherapy. Standard error bars were used. Linear mixed model test $(p=0.003$ and $p=0.006$ for the probiotic and probiotic plus honey groups respectively).

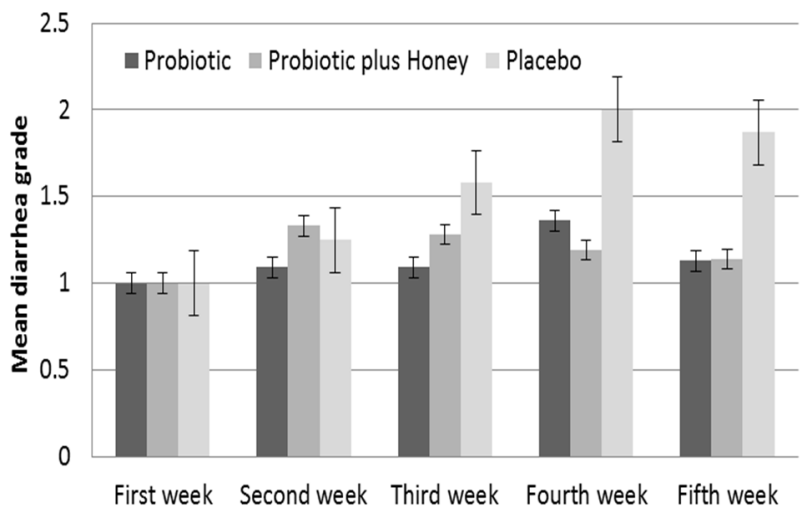

Figure 2. Comparison of the mean diarrhea grade per week during pelvic radiotherapy. Standard error bars were used. Mann-Whitney test ( $p=0.007$ and 0.001 for probiotic and $p<$ 0.001 and $p=0.001$ for probiotic plus honey in weeks 4 and 5 respectively). 


\section{Stool consistency}

The mean stool consistency was initially 3.8 for all groups. During the treatment, mean consistency stool was 4.3 for the probiotic and probiotic plus honey groups and 5.7 for the placebo group. The results of Mann-Whitney test showed that the mean stool consistency score in the probiotic and the probiotic plus honey groups were significantly lower than the placebo group in weeks 4 and 5 of treatment $(\mathrm{p}=0.003$ and 0.004 for the probiotic group and $p<0.001$ and $p=0.005$ for the probiotic plus honey group in weeks 4 and 5 respectively) (figure 3 ).

\section{The need for antidiarrheal medication}

During pelvic radiotherapy was found that antidiarrheal medication is required for 13 (19.4\%) patients. Among all patients, 9.1\%, $9.5 \%$ and $37.5 \%$ belonged to the probiotic, probiotic plus honey and placebo groups respectively needed for antidiarrheal medication. In the first week no patients needed for antidiarrheal medication. Only one and two patients in the fifth week needed for antidiarrheal medication in the probiotic and probiotic plus honey respectively. The number of patients who needed for antidiarrheal medication in the placebo group was one, four, three, one in weeks 2, 3, 4, 5 respectively. The Fisher's exact test showed that the number of patients who needed to antidiarrheal medication

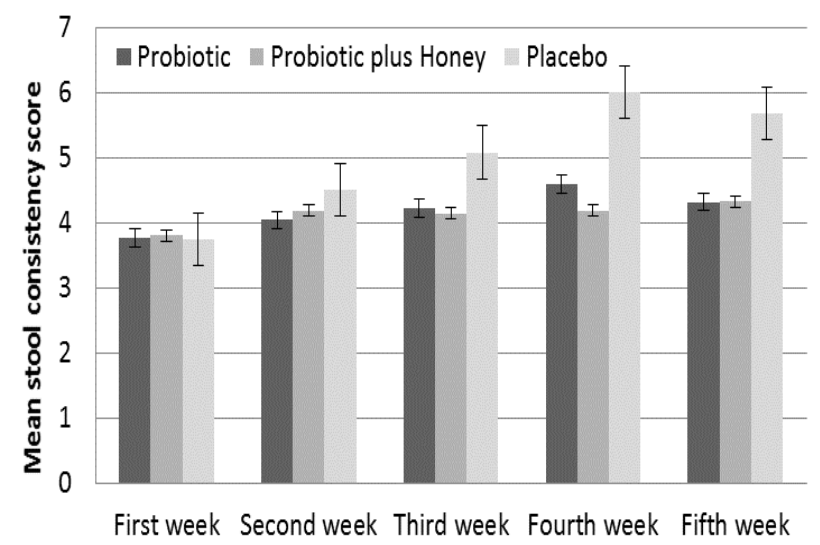

Figure 3. Comparison of the mean stool consistency score per week during pelvic radiotherapy. Standard error bars were used. Mann-Whitney test $(p=0.003$ and 0.004 for the probiotic group and $p<0.001$ and $p=0.005$ for the probiotic plus honey group in weeks 4 and 5 respectively). in the placebo group was significantly higher than the probiotic $(\mathrm{P}=0.038)$ and probiotic plus honey groups $(\mathrm{P}=0.04)$ (figure 4$)$. The result of Fisher's exact test also indicated that patients in the groups that received probiotic used antidiarrheal medication later than the placebo group $(\mathrm{P}=0.021$ and $\mathrm{P}=0.041$ for the probiotic and probiotic plus honey respectively).

\section{Adverse effects}

Some of probiotics users reported stomach pain and bloating. During pelvic radiotherapy, three patients (they belonged to probiotic user; with or without honey) complained of upper abdominal pain. The causal link between the complaint and the probiotic was not investigated. During the treatment 45 patients complained of bloating (19 patients of the probiotic group, 16 patients of the probiotic plus honey group and 10 patients in the placebo group). The results of the Chi-square test showed that the number of patients with bloating in the probiotic groups (alone or plus honey) was significantly higher than the placebo group $(\mathrm{P}=0.002$ and 0.021 for the probiotic and the probiotic plus honey groups, respectively) (figure 5).

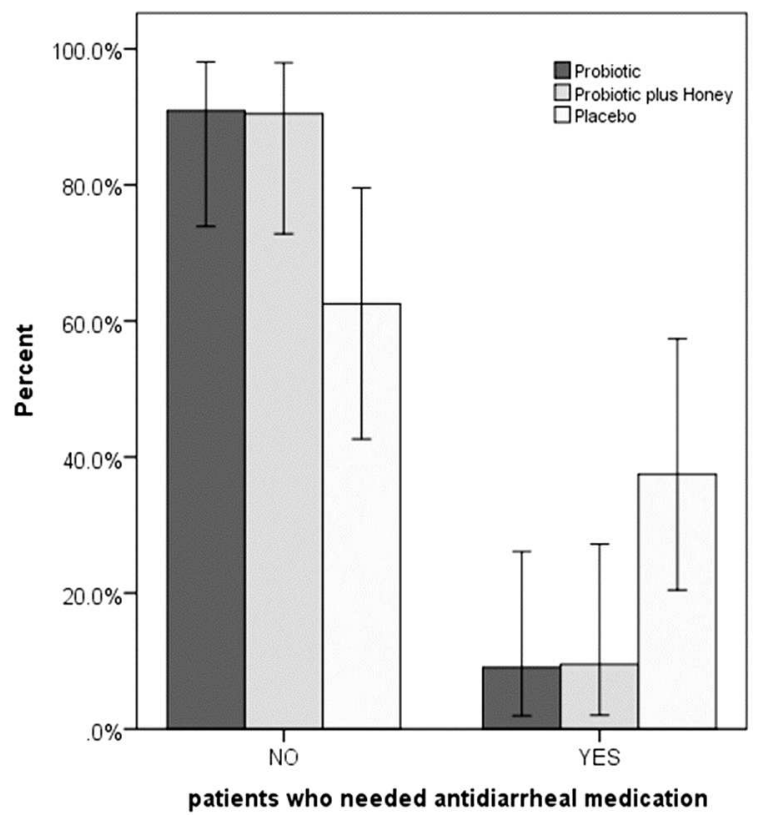

Figure 4. Comparison of patients who needed antidiarrheal medication and those who did not during pelvic radiotherapy. Fisher's exact test $(\mathrm{P}=0.038$ and $\mathrm{P}=0.04$ for the probiotic and probiotic plus honey groups, respectively).

Int. J. Radiat. Res., Vol. 14 No. 3, July 2016 


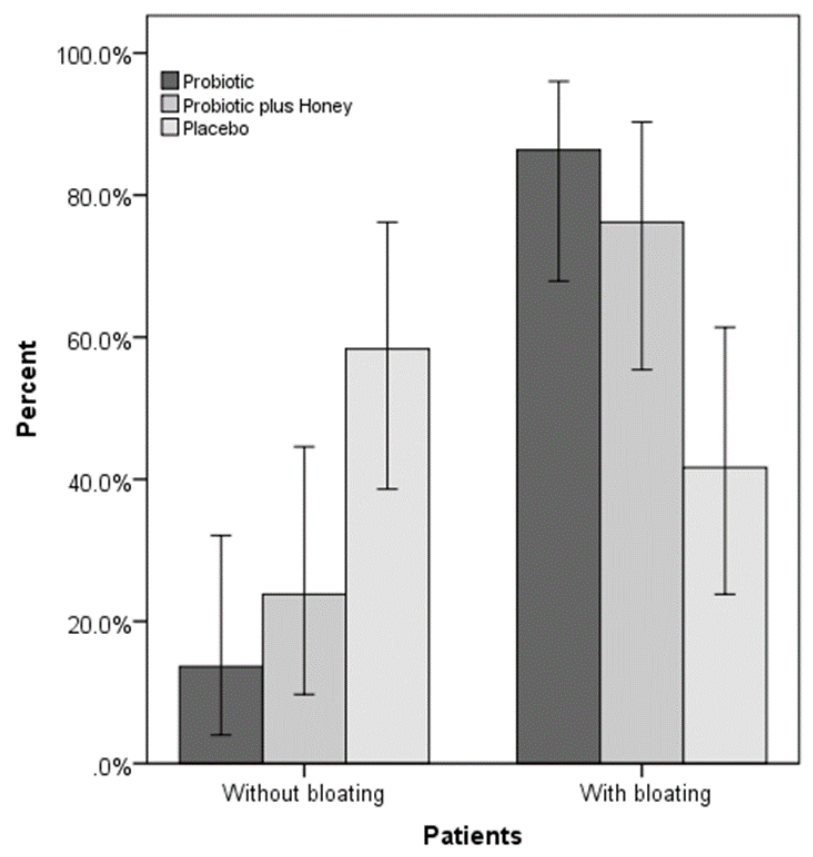

Figure 5. Comparison of patients with and without bloating during pelvic radiotherapy. Chi-square test ( $\mathrm{P}=0.002$ and 0.021 for the probiotic and the probiotic plus honey groups, respectively).

\section{DISCUSSION}

Diarrhea is most (80\%) and worst side effect of pelvic radiotherapy ${ }^{(1-3)}$. Different studies have focused on the prevention or control of radiation -induced diarrhea. In the current study probiotic with or without honey were used in patients undergoing pelvic radiotherapy.

Some studies show significant beneficial effects of probiotics on control of radiationinduced diarrhea; Delia et al. $(19,51)$ and Timko (31). The benefits of probiotic therapy with VSL\#3 (probiotics, including: L. casei, L. plantarum, $L$. acidophilus, L.delbruekii subsp. Bulgaricus, B. longum, B. breve, and B. infantis, Streptococcus salivarius subsp. Thermophiles) have been recorded in 490 patients during radiotherapy after surgery for the abdominal and pelvic cancer in a double-blind, placebo- controlled trial. The mean daily number of bowel movements in these 490 patients was $4.6 \pm 2$ and $12.3 \pm 4$ in VSL\#3 and placebo recipients, respectively (19). Similarly, a randomized study has demonstrated a decrease in the mean number of bowel movements $(\mathrm{p}<0.05)$ and $a$ decrease in the incidence of diarrhea $(p<0.01)$ in patients undergoing pelvic radiotherapy and taking Lactobacillus acidophilus (29). Another randomized placebo controlled nutrition trial reported that yogurt containing Lactobacillus casei DN-114 001 significantly improved the consistency of stool, without reducing the incidence of radiation-induced diarrhea (21).

The mean of bowel movement frequency in our study was 2.09 and 2.18 per day in the probiotic and probiotic plus honey groups respectively and 3.32 in the placebo group, which is obviously lower to the 5.1 and 14.7 per day in the VSL\#3 and placebo groups respectively reported by Delia et al. $(19,51)$, but results of Timko et al. study (4.16 in the probiotic group and 2.52 in the Hylak Tropfen Forte group) (31) were closer to our study. Some investigators considered stool consistency, rather than frequency of bowel movement, to be a more appropriate measure of diarrhea (52). In this study, the stool consistency was normal (score 4.3 in Bristol scale) for the probiotic and probiotic plus honey groups and soft (score ` 5.7 in Bristol scale) for the placebo group, which is obviously lower than stool consistency score in the probiotic (taking liquid yogurt containing $L$. casei DN-114 001 at $10^{8} \mathrm{CFU} / \mathrm{g}$ ) and placebo groups in Giralt et al. study (21). In Giralt et al. 
study over $80 \%$ of patients undergoing pelvic radiotherapy reached the worse stool consistency score (6 and 7 in Bristol scale), without significant difference between the two (probiotic and placebo) groups(21). Since some studies show that yogurt is effective in the treatment of diarrhea (47) and it is as probiotic carrier food (48), low-fat yogurt taking in our study may be a reason for the difference of the number of bowel movements and stool consistency score between this study and similar studies $(19,53)$ in the patients who underwent pelvic radiotherapy. More controlled clinical trials are needed to confirm this issue.

In the present study patients who need for antidiarrheal medication in the probiotic and probiotic plus honey groups were significantly less than the placebo group, also in Timko et al. study $27 \%$ in the probiotic group and 55\% in the Hylak Tropfen Forte group needed for antidiarrheal medication (31). The first prescription of antidiarrheal medication in the Delia et al. study (19) was in the VSL\#3 group significantly later than in the control group, which this result was similar to our study. Although we expected probiotic plus honey is more effective than probiotic alone, but statistically significant difference was not found. This finding may have resulted from effect of honey as prebiotic needed prolonged time to improve probiotics. No similar study has been found.

Important differences between our study and other studies are the treatment protocols, onset of intake as well as dosages, species and strains of probiotics. The most commonly used strains of probiotics in reported researchs are lactobacillus, Bifidobacterium and cocci $(22,23,25$, 36). Since bloating may be a side effect of probiotics use (39), in this study bloating was asked the patients. These results showed that patients taking probiotics suffered from bloating more than the control group during the treatment. However, the severity of the symptom was not enough to discontinue the use of probiotics. In the similar studies that have investigated effects of probiotics on diarrhea, bloating has not evaluated.

Our result showed that probiotics improved the quality of life of patients undergoing pelvic radiation. So probiotic usage can prevent premature discontinuation of pelvic radiation and increase radiation dose if need be. The verification of beneficial use of probiotics in patients undergoing radiation needs more clinical trial. Since probiotics (with or without honey) did not completely prevent moderate to severe diarrhea during treatment, this study suggest that probiotics are used as a beneficial supplement to reduce pelvic radiation-induced diarrhea.

\section{ACKNOWLEDGMENT}

The authors thanks to Zist Takhmir Company officials for providing probiotics and placebo, Mr. Hadi; Allas Company management for providing the yogurt, Keshtzare Sabz Company management for providing honey, Radiation Oncology Clinic of Sayyed-Al-Shohada Hospital staff also and the Lab Manager of Food and drug Administration Isfahan University of Medical Sciences. This research was supported by a grant from the University of Isfahan and Cancer Prevention Research Center at Isfahan University of Medical Sciences (Project No: 291196).

\section{Conflict of interest: Declared None}

\section{REFERENCES}

1. Andreyev J (2005) Gastrointestinal complications of pelvic radiotherapy: are they of any importance? Gut, 54(8): 1051-4.

2. McGough C, Baldwin C, Frost G, Andreyev H (2004) Role of nutritional intervention in patients treated with radiotherapy for pelvic malignancy. British Journal of Cancer, 90(12): 2278-87.

3. Abayomi J, Kirwan J, Hackett A, Bagnall G (2005) A study to investigate women's experiences of radiation enteritis following radiotherapy for cervical cancer. Journal of Human Nutrition and Dietetics, 18(5): 353-63.

4. Fuccio L, Guido A, Eusebi LH, Laterza L, Grilli D, Cennamo V, et al. (2009) Effects of probiotics for the prevention and treatment of radiation-induced diarrhea. Journal of Clinical Gastroenterology, 43(6): 506-13.

5. Gami B, Harrington K, Blake P, Dearnaley D, Tait D, Davies J, et al. (2003) How patients manage gastrointestinal

Int. J. Radiat. Res., Vol. 14 No. 3, July 2016 


\section{Mansouri-Tehrani et al. / Probiotics and radiation-induced diarrhea}

symptoms after pelvic radiotherapy. Alimentary Pharmacology \& Therapeutics, 18(10): 987-94.

6. Hovdenak N, Fajardo LF, Hauer-Jensen M (2000) Acute radiation proctitis: a sequential clinicopathologic study during pelvic radiotherapy. International Journal of Radiation Oncology Biology Physics, 48(4):1111-7.

7. Mohagheghi $F$ and Mohammadi M (2008) Innovation of a method to decrease interruption time during treatment of pelvic area carcinomas. International Journal of Radiation Research, 5(4): 175-80.

8. Savard J and Sawatzky JA (2007) The use of a nursing model to understand diarrhea and the role of probiotics in patients with inflammatory bowel disease. Gastroenterology Nursing, 30(6): 418-23.

9. Larsen A, Bjørge B, Klementsen B, Helgeland L, WentzelLarsen T, Fagerhol MK, et al. (2007) Time patterns of changes in biomarkers, symptoms and histopathology during pelvic radiotherapy. Acta Oncologica, 46(5): 639-50.

10. Blanarova C, Galovicova A, Petrasova D (2008) Use of probiotics for prevention of radiation-induced diarrhea. Bratislavske lekarske listy, 110(2): 98-104.

11. Gibson RJ and Keefe DM (2006) Cancer chemotherapyinduced diarrhoea and constipation: mechanisms of damage and prevention strategies. Supportive Care in Cancer, 14(9): 890-900.

12. Webb GJ, Brooke R, De Silva AN (2013) Chronic radiation enteritis and malnutrition. Journal of Digestive Diseases, 14(7): 350-7.

13. Zimmerer T, Böcker U, Wenz F, Singer M (2008) Medical prevention and treatment of acute and chronic radiation induced enteritis-is there any proven therapy? A short Review. Zeitschrift für Gastroenterologie, 46(05): 441-8.

14. Gibson RJ, Keefe DM, Lalla RV, Bateman E, Blijlevens N, Fijlstra M, et al. (2013) Systematic review of agents for the management of gastrointestinal mucositis in cancer patients. Supportive Care in Cancer, 21(1): 313-26.

15. Quigley EM and Quera R (2006) Small intestinal bacterial overgrowth: roles of antibiotics, prebiotics, and probiotics. Gastroenterology, 130(2): S78-S9O.

16. Keefe DM, Schubert MM, Elting LS, Sonis ST, Epstein JB, Raber-Durlacher JE, et al. (2007) Updated clinical practice guidelines for the prevention and treatment of mucositis. Cancer, 109(5): 820-31.

17. Wedlake L, Shaw C, Whelan K, Andreyev H (2013) Systematic review: the efficacy of nutritional interventions to counteract acute gastrointestinal toxicity during therapeutic pelvic radiotherapy. Alimentary Pharmacology \& Therapeutics, 37(11): 1046-56.

18. Ravasco P, Monteiro-Grillo I, Vidal PM, Camilo ME (2005) Dietary counseling improves patient outcomes: a prospective, randomized, controlled trial in colorectal cancer patients undergoing radiotherapy. Journal of Clinical Oncology, 23(7): 1431-8.

19. Delia P, Sansotta G, Donato V, Frosina P, Messina G, De Renzis C, et al. (2007) Use of probiotics for prevention of radiation-induced diarrhea. World Journal of Gastroenterology, 13(6): 912-5.
20. Muehlbauer PM, Thorpe D, Davis A, Drabot R, Rawlings BL, Kiker E (2009) Putting evidence into practice. Clinical Journal of Oncology Nursing, 13(3):336-41.

21. Giralt J, Regadera JP, Verges R, Romero J, de la Fuente I, Biete A, et al. (2008) Effects of probiotic lactobacillus Casei DN-114 001 in prevention of radiation-induced diarrhea: Results from multicenter, randomized, Placebo-Controlled nutritional trial. International Journal of Radiation Oncology Biology Physics, 71(4): 1213-9.

22. Ciorba MA (2012) A gastroenterologist's guide to probiotics. Clinical Gastroenterology and Hepatology, 10 (9): 960-8.

23. Ringel Y, Quigley EM, Lin HC (2012) Using probiotics in gastrointestinal disorders. The American Journal of Gastroenterology Supplements, 1(1): 34-40.

24. Ritchie ML and Romanuk TN (2012) A meta-analysis of probiotic efficacy for gastrointestinal diseases. PLoS One, 7 (4): e34938, 1-11.

25. Sanders ME, Guarner F, Guerrant R, Holt PR, Quigley EM, Sartor RB, et al. (2013) An update on the use and investigation of probiotics in health and disease. Gut, 62 (5): 787-96.

26. Famularo G, De Simone C, Matteuzzi D, Pirovano F (1999) Traditional and high potency probiotic preparations for oral bacteriotherapy. Biodrugs, 12(6): 455-70.

27. Famularo G, Mosca L, Minisola G, Trinchieri V, Simone CD (2003) Probiotic lactobacilli: a new perspective for the treatment of inflammatory bowel disease. Current Pharmaceutical Design, 9(24): 1973-80.

28. Chitapanarux I, Chitapanarux T, Traisathit P, Kudumpee $S$, Tharavichitkul E, Lorvidhaya V (2010) Research Randomized controlled trial of live lactobacillus acidophilus plus bifidobacterium bifidum in prophylaxis of diarrhea during radiotherapy in cervical cancer patients. Radiation Oncology, 5:31-6.

29. Salminen E, Elomaa I, Minkkinen J, Vapaatalo H, Salminen $S$ (1988) Preservation of intestinal integrity during radiotherapy using live Lactobacillus acidophilus cultures. Clinical Radiology, 39(4): 435-7.

30. Urbancsek H, Kazar T, Mezes I, Neumann K (2001) Results of a double-blind, randomized study to evaluate the efficacy and safety of Antibiophilus ${ }^{\circledR}$ in patients with radiation-induced diarrhoea. European Journal of Gastroenterology \& Hepatology, 13(4): 391-6.

31. Timko J (2010) Probiotics as prevention of radiationinduced diarrhoea. Journal of Radiotherapy in Practice, 9 (04): 201-8.

32. Österlund $P$, Ruotsalainen $T$, Korpela R, Saxelin M, Ollus $A$, Valta $P$, et al. (2007) Lactobacillus supplementation for diarrhoea related to chemotherapy of colorectal cancer: a randomised study. British Journal of Cancer, 97(8): 102834.

33. Reid G, Sanders M, Gaskins HR, Gibson GR, Mercenier A, Rastall $R$, et al. (2003) New scientific paradigms for probiotics and prebiotics. Journal of Clinical Gastroenterology, 37(2): 105-18. 
34. Guarner F and Schaafsma G (1998) Probiotics. International journal of food microbiology, 39(3): 237-8.

35. Schrezenmeir J and de Vrese M (2001) Probiotics, prebiotics, and synbiotics-approaching a definition. The American Journal of Clinical Nutrition, 73(2): 361s-4s.

36. Gorbach S (2002) Probiotics in the third millennium. Digestive and Liver Disease, 34: S2-S7.

37. Rijkers GT, Bengmark S, Enck P, Haller D, Herz U, Kalliomaki M, et al. (2010) Guidance for substantiating the evidence for beneficial effects of probiotics: current status and recommendations for future research. The Journal of Nutrition, 140(3): 671S-6S.

38. Demers M, Dagnault A, Desjardins J (2014) A randomized double-blind controlled trial: Impact of probiotics on diarrhea in patients treated with pelvic radiation. Clinical Nutrition, 33(5): 761-7.

39. Marteau P and Seksik P(2004) Tolerance of probiotics and prebiotics. Journal of Clinical Gastroenterology, 38: S67S9.

40. Nagpal R and Kaur A (2011) Synbiotic effect of various prebiotics on in vitro activities of probiotic lactobacilli. Ecology of Food and Nutrition, 50(1): 63-8.

41. Al-Waili N, Salom K, Al-Ghamdi AA (2011) Honey for wound healing, ulcers, and burns; data supporting its use in clinical practice. The Scientific World Journal, 11: 76687.

42. Parmar J, Hunjan P, Brown A, Telfer M (2013) Honey dressing use for the management of split thickness skin graft donor sites: a technical note. British Journal of Oral and Maxillofacial Surgery, 51(3): e40-e1.

43. Yaghoobi N, Al-Waili N, Ghayour-Mobarhan M, Parizadeh S, Abasalti Z, Yaghoobi Z, et al. (2008) Natural honey and cardiovascular risk factors; effects on blood glucose, cholesterol, triacylglycerole, CRP, and body weight compared with sucrose. The Scientific World Journal, 8: 463-9.
44. Al-Waili NS, Salom K, Butler G, Al Ghamdi AA (2011) Honey and microbial infections: a review supporting the use of honey for microbial control. Journal of Medicinal Food, 14 (10): 1079-96.

45. Bogdanov S (2009) Nutritional and functional properties of honey. Voprosy Pitaniia, 79(6): 4-13.

46. Chow J (2002) Probiotics and prebiotics: a brief overview. Journal of Renal Nutrition, 12(2): 76-86.

47. Shan NP (2006) Health benefits of yogurt and fermented milks. Manufacturing yogurt and fermented milks, Blackwell Publishing, Oxford, UK. 327-40.

48. Lourens-Hattingh A and Viljoen BC (2001) Yogurt as probiotic carrier food. International Dairy Journal, 11(1): 117.

49. U.S. National Institutes of Health, National Cancer Institute. Common Toxicity Criteria, version 2.0. Bethesda: National Institutes of Health, National Cancer, Institute; 1999. Available from: ctep.cancer.gov. Accessed April 30, 1999.

50. O'Donnell L, Virjee J, Heaton KW (1990) Detection of pseudodiarrhoea by simple clinical assessment of intestinal transit rate. BMJ: British Medical Journal, 300 (6722): 439.

51. Delia P, Sansotta G, Donato V, Messina G, Frosina P, Pergolizzi S, et al. (2002) Prophylaxis of diarrhoea in patients submitted to radiotherapeutic treatment on pelvic district: personal experience. Digestive and Liver Disease, 34: S84-S6.

52. Longstreth GF, Thompson WG, Chey WD, Houghton LA, Mearin F, Spiller RC (2006) Functional bowel disorders. Gastroenterology, 130(5): 1480-91.

53. Giralt J, Regadera JP, Verges R, Romero J, de la Fuente I, Biete A, et al. (2008) Effects of probiotic Lactobacillus casei DN-114 001 in prevention of radiation-induced diarrhea: results from multicenter, randomized, placebo-controlled nutritional trial. International Journal of Radiation Oncology Biology Physics, 71(4): 1213-9. 
